\title{
CONE LATTICES OF UPPER SEMICONTINUOUS FUNCTIONS
}

\author{
GERALD BEER
}

\begin{abstract}
Let $X$ be a compact metric space. A well-known theorem of $M$. H. Stone states that if $\Omega$ is a vector lattice of continuous functions on $X$ that separates points and contains a nonzero constant function, then the uniform closure of $\Omega$ is $C(X)$. In this article we generalize Stone's sufficient conditions to the upper semicontinuous functions on $X$ topologized in a natural way.
\end{abstract}

Let $X$ be a compact metric space with metric $d$ and let $C(X)$ denote the real valued continuous functions on $X$, made a normed linear space by defining the norm of each $f$ in $C(X)$ by the formula $\|f\|=\sup _{x \in X}|f(x)|$. Dense sublattices $\Omega$ of $C(X)$ are characterized by the Stone Approximation Theorem [5]: $\Omega$ is dense if and only if for each $\epsilon>0$, for each $\left(x_{1}, x_{2}\right)$ in $X \times X$, and for each $f$ in $C(X)$, there exists $h$ in $\Omega$ such that $\left|h\left(x_{1}\right)-f\left(x_{1}\right)\right|<\epsilon$ and $\left|h\left(x_{2}\right)-f\left(x_{2}\right)\right|<\epsilon$. If one knows that, in addition, $\Omega$ is a vector space, then the following conditions are sufficient for $\Omega$ to be dense: $\Omega$ separates points and contains a nonzero constant function.

In [2] this author generalized the purely lattice theoretic Stone Approximation Theorem to the lattice of real valued upper semicontinuous functions $U C(X)$ on a compact metric space $X$ by viewing $U C(X)$ as a subspace of the hyperspace of $X \times R$ : if $h \in U C(X)$ we identify $h$ with its hypograph

$$
\text { hypo } h=\{(x, \alpha): \alpha \leq h(x)\}
$$

a closed subset of $X \times R$. Choosing a metric compatible with the product uniformity on $X \times R\left(\rho\left[\left(x_{1}, \alpha_{1}\right),\left(x_{2}, \alpha_{2}\right)\right]=\max \left\{d\left(x_{1}, x_{2}\right),\left|\alpha_{1}-\alpha_{2}\right|\right\}\right.$ is computationally the simplest), we define the distance $D$ between two u.s.c. functions to be the Hausdorff distance between their hypographs. $D$-convergence for u.s.c. functions can be described without reference to the metric $D$ : a sequence $\left\{f_{n}\right\}$ in $U C(X)$ $D$-converges to $f$ if and only if, for each $x$ in $X$,

(i) whenever $\left\{x_{n}\right\} \rightarrow x$ then $\lim \sup _{n \rightarrow \infty} f_{n}\left(x_{n}\right) \leq f(x)$;

(ii) there exists a sequence $\left\{x_{n}\right\} \rightarrow x$ such that $\lim _{n \rightarrow \infty} f_{n}\left(x_{n}\right)=f(x)$.

In the context of compact spaces $D$-convergence for u.s.c. functions is dual to the notion of infimal convergence for lower semicontinuous functions introduced by Wijsman [6], later termed $\Gamma^{-}$convergence by De Giorgi and Franzoni [3], and now called epi-convergence [4].

DEFinition. A class $\Omega$ of real valued functions on a compact metric space $X$ is said to isolate points in $X \times R$ if whenever $\left(x_{1}, \alpha_{1}\right)$ and $\left(x_{2}, \alpha_{2}\right)$ are points in $X \times R$ such that either $x_{1} \neq x_{2}$ or $x_{1}=x_{2}$ and $\alpha_{1}<\alpha_{2}$ then there exists $h$ in $\Omega$ such that $\left(x_{1}, \alpha_{1}\right) \in$ int hypo $h$ and $\alpha_{2}>h\left(x_{2}\right)$.

Received by the editors July 31, 1981 and, in revised form, December 7, 1981.

1980 Mathematics Subject Classification. Primary 26A15, 41A65, 54B20.

Key words and phrases. Semicontinuous function, Stone Approximation Theorem, Hausdorff metric, monotone functional.

(C) 1982 American Mathematical Society 0002-9939/82/0000-0189/\$02.00 
Definition. A class $\Omega$ of u.s.c. functions on a compact metric space $X$ is said to be upper dense in $U C(X)$ if for each $f \in U C(X)$ and each $\epsilon>0$ there exists $h \in \Omega$ satisfying $h \geq f$ and $D(h, f)<\epsilon$.

The main result of [2] can now be stated; we note that the "if" implication is actually proved in [1].

THEOREM A. Let $X$ be a compact metric space and let $\Omega$ be a sublattice of $U C(X)$. Then $\Omega$ isolates points in $X \times R$ if and only if $\Omega$ is upper dense in $U C(X)$.

If $\Omega$ is upper dense, then since $\Omega$ is a lattice, each $f$ in $U C(X)$ is the $D$-limit of a decreasing sequence of functions in $\Omega$. For decreasing sequences of u.s.c. functions, $D$-convergence is equivalent to pointwise convergence. From this fact, the Stone Approximation Theorem easily follows from Theorem A and Dini's Theorem.

We now generalize the vector lattice sufficient conditions to $U C(X)$. Unfortunately, $U C(X)$ is not a vector space; however, it is a cone, i.e., it is closed under addition and under multiplication by nonnegative scalars. To state our conditions in the simplest form we mention an alternative expression of membership to the interior of the hypograph of a function. If $f: X \rightarrow R$ we define the lower envelope $\underline{f}: X \rightarrow[-\infty, \infty)$ of $f$ by the formula $\underline{f}(x)=\sup _{\delta>0} \inf \left\{f(y): y \in B_{\delta}[x]\right\}$ where $\bar{B}_{\delta}[x]$ denotes the closed ball of radius $\delta$ about $x$. We leave it to the reader to verify the following facts:

(i) $\underline{f}(x)=f(x)$ if and only if $f$ is lower semicontinuous at $x$;

(ii) $(x, \alpha) \in$ int hypo $f$ if and only if $\alpha<\underline{f}(x)$.

THEOREM 1. Let $X$ be a compact metric space and let $\Omega$ be a cone sublattice of $U C(X)$. Suppose that $\Omega$ contains the constant functions and for each $(a, b)$ in $X \times X$ where $a \neq b$ there exists $h \in \Omega$ such that $\underline{h}(a)>h(b)$. Then $\Omega$ is $D$-upper dense in $U C(X)$.

ProOF. We need only show that $\Omega$ isolates points in $X \times R$. We consider three cases for points $\left(x_{1}, \alpha_{1}\right)$ and $\left(x_{2}, \alpha_{2}\right)$ in the product: (i) $x_{1}=x_{2}$ and $\alpha_{1}<\alpha_{2}$, (ii) $x_{1} \neq x_{2}$ and $\alpha_{2}<\alpha_{1}$, (iii) $x_{1} \neq x_{2}$ and $\alpha_{2} \geq \alpha_{1}$.

Case (i). The constant function $h=\frac{1}{2}\left(\alpha_{1}+\alpha_{2}\right)$ isolates $\left(x_{1}, \alpha_{1}\right)$ from $\left(x_{2}, \alpha_{2}\right)$.

Case (ii). Choose $h \in \Omega$ such that $\underline{h}\left(x_{1}\right)>h\left(x_{2}\right)$. By the definition of the lower envelope there exists $\delta>0$ such that

$$
\alpha=\inf \left\{h(y): y \in B_{\delta}\left[x_{1}\right]\right\}>h\left(x_{2}\right) .
$$

Since $\Omega$ is closed under addition and under multiplication by nonnegative scalars

$$
g=\frac{\alpha_{1}-\alpha_{2}+2}{\alpha-h\left(x_{2}\right)} \cdot(h-\alpha)
$$

belongs to $\Omega$. Clearly $g$ is nonnegative on $B_{\delta}\left[x_{1}\right]$ and $g\left(x_{2}\right)=\alpha_{2}-\alpha_{1}-2$. Set $v=g+\alpha_{1}+1$. Then $v\left(x_{2}\right)=\alpha_{2}-1$ and for each $y$ in $B_{\delta}\left[x_{1}\right] v(y) \geq \alpha_{1}+1$. It follows that $v \in \Omega$ and that $v$ isolates $\left(x_{1}, \alpha_{1}\right)$ from $\left(x_{2}, \alpha_{2}\right)$.

Case (iii). Let $h, \delta$, and $\alpha$ have the same meaning as in case (ii). Then $g=$ $h+\alpha_{1}-\frac{1}{2}\left[\alpha+h\left(x_{2}\right)\right]$ is in $\Omega$. Moreover, $g\left(x_{2}\right)=\alpha_{1}-\frac{1}{2}\left[\alpha-h\left(x_{2}\right)\right]<\alpha_{1} \leq \alpha_{2}$ and for each $y \in B_{\delta}\left[x_{1}\right]$,

$$
g(y) \geq \alpha+\alpha_{1}-\frac{1}{2}\left[\alpha+h\left(x_{2}\right)\right]>\alpha_{1} .
$$

Thus $g$ isolates $\left(x_{1}, \alpha_{1}\right)$ from $\left(x_{2}, \alpha_{2}\right)$. 
By the remarks following Theorem A, Stone's vector lattice sufficient conditions immediately fall out of Theorem 1 . The details are left to the reader.

THEOREM 2. Let $X$ be a compact metric space and let $\Omega$ be a cone sublattice of $U C(X)$. Suppose that $\Omega$ contains the constant functions, and each D-u.s.c. monotone positively homogeneous functional $\phi: \Omega \rightarrow R$ has a unique extension $\phi^{*}: U C(X) \rightarrow$ $[-\infty, \infty)$ with the same properties. Then $\Omega$ is D-upper dense in $U C(X)$.

Proof. Suppose $\Omega$ is not $D$-upper dense. Since $C(X)$ is $D$-upper dense, there exists a continuous function $f$ which cannot be $D$-approximated from above by members of $\Omega$. Since $\Omega$ contains the constants and the Hausdorff distance $D$ between hypographs defined in terms of the metric on $X \times R$ described earlier is invariant under vertical translation, we can assume that $f \geq 1$. Choose $\lambda>0$ such that for each $h \in \Omega, h \geq f$ implies that $D(h, f)>\lambda$. For each $h \in U C(X)$ set

$$
\begin{aligned}
& F(h)=\{\alpha>0: h / \alpha \geq f\}, \\
& E(h)=\{\alpha>0: h / \alpha \geq f \text { and } \exists z \in X \text { such that } h(z) / \alpha \geq f(z)+\lambda\} .
\end{aligned}
$$

For each $h \in U C(X), E(h) \subseteq F(h)$; moreover, the following are equivalent: (i) $E(h) \neq \emptyset$, (ii) $F(h) \neq \emptyset$, (iii) $\inf \{h(x): x \in X\}>0$. Whenever $E(h)$ is nonempty the upper semicontinuity of $h$, the continuity of $f$, and the compactness of $X$ together imply that $\sup E(h) \in E(h)$. Thus, we may define the following functional $\psi: U C(X) \rightarrow R:$

$$
\psi(h)= \begin{cases}\max E(h) & \text { if } E(h) \neq \emptyset \\ 0 & \text { otherwise. }\end{cases}
$$

Clearly $\psi$ is both monotone and positively homogeneous. To show that $\psi$ is $D$-u.s.c., we show that for each $\alpha, \psi^{-1}([\alpha, \infty))$ is $D$-closed. This is trivial for $\alpha \leq 0$. If $\alpha>0$ let $\left\{h_{n}\right\}$ be a sequence in $\psi^{-1}([\alpha, \infty)), D$-convergent to an u.s.c. function $h$. Then (i) for each $x \in X$ and for each $n \in Z^{+},(1 / \alpha) h_{n}(x) \geq f(x)$, (ii) for each $n \in Z^{+}$there exists $z_{n} \in X$ such that $(1 / \alpha) h_{n}\left(z_{n}\right) \geq f\left(z_{n}\right)+\lambda$. We now apply the convergence class description of the $D$-topology. At each point $x$,

$$
\frac{h(x)}{\alpha} \geq \limsup _{n \rightarrow \infty} \frac{h_{n}(x)}{\alpha} \geq f(x) .
$$

By passing to a subsequence we can assume $\left\{z_{n}\right\}$ converges to some point $z$. Since $f \in C(X)$ we have

$$
\frac{h(z)}{\alpha} \geq \limsup _{n \rightarrow \infty} \frac{h_{n}\left(z_{n}\right)}{\alpha} \geq \limsup _{n \rightarrow \infty} f\left(z_{n}\right)+\lambda=f(z)+\lambda .
$$

This proves that $\psi$ is $D$-u.s.c. It is even easier to see that $\sigma: U C(X) \rightarrow R$, defined by

$$
\sigma(h)= \begin{cases}\max F(h) & \text { if } F(h) \neq \emptyset, \\ 0 & \text { otherwise, }\end{cases}
$$

is monotone, positively homogeneous, and $D$-u.s.c.

Now if $h \in \Omega$ and $h / \alpha \geq f$, the fact that

$$
\sup _{x \in X}\left|\frac{h}{\alpha}(x)-f(x)\right| \geq D\left(\frac{h}{\alpha}, f\right)>\lambda
$$

implies the existence of $z \in X$ such that $(1 / \alpha) h(z) \geq f(z)+\lambda$. Hence, if $h \in \Omega$ and $E(h) \neq \emptyset$, we have $E(h)=F(h)$. As a result $\psi|\Omega=\sigma| \Omega$. We have thus 
produced a monotone positively homogeneous $D$-u.s.c. functional on $\Omega$ with two different extensions, for $\sigma(f)=1$ whereas $\psi(f)<1$.

Two remarks are in order. First, the condition on the extension of functionals on $\Omega$ is also necessary for $\Omega$ to be $D$-upper dense. This follows from Theorem 4 of [1] which shows the "first Daniell extension" of any monotone $D$-u.s.c. functional $\phi$ on a $D$-upper dense sublattice of $U C(X)$ to be the unique monotone $D$-u.s.c. extension of $\phi$ to $U C(X)$. Clearly, if $\phi$ is positively homogeneous, so is the Daniell extension. Second, we cannot conclude from the proof of Theorem 2 that the ability to extend $D$-continuous monotone positively homogeneous functionals, on a cone sublattice $\Omega$ that contains the constants, forces the cone sublattice to be $D$-upper dense. We cannot expect the functional $\sigma$ defined in the proof to be $D$-continuous. For example, suppose $f \in C[0,1]$ and $f \geq 1$. Let $h_{n}$ be the function whose graph consists of two line segments, one joining $(0,0)$ to $(1 / n, 1)$; the other joining $(1 / n, 1)$ to $(1,1)$. Although $\left\{h_{n}\right\} D$-converges to 1 , for each $n, \sigma\left(h_{n}\right)=0$; whereas $\sigma(1)=$ $[\sup \{f(x): 0 \leq x \leq 1\}]^{-1}>0$.

We close with a new density theorem for $C(X)$ with respect to the norm topology.

THEOREM 3. Let $X$ be a compact metric space. Let $\Omega$ be a cone sublattice of $C(X)$ that contains the constant functions. Then $\Omega$ is dense in $C(X)$ with respect to the norm topology if and only if each monotone positively homogeneous functional $\phi: \Omega \rightarrow R$ that is continuous with respect to the norm topology has a unique monotone positively homogeneous $D$-u.s.c. extension $\phi^{*}: U C(X) \rightarrow[-\infty, \infty)$.

Proof. If $\Omega$ is dense in $C(X)$ in the usual sense, then since $C(X)$ is $D$-upper dense in $U C(X)$ and the norm topology on $C(X)$ (or $U C(X)$ ) is stronger than the $D$-topology, $\Omega$ must be $D$-upper dense in $U C(X)$. Since $\Omega \subseteq C(X)$, by Theorem 5 of [1], a monotone functional $\phi: \Omega \rightarrow R$ is u.s.c. with respect to the norm topology if and only if it is $D$-u.s.c. The necessity of the condition now follows from Theorem 4 of [1] cited previously. Conversely, if $\Omega$ is not dense in $C(X)$ with the norm topology, it is not $D$-upper dense in $U C(X)$. Using the construction in the proof of Theorem 2 the functional $\sigma \mid \Omega$ is a continuous functional with respect to the norm topology (but not with respect to the $D$-topology) that admits more than one $D$-u.s.c. extension to $U C(X)$.

\section{BIBLIOGRAPHY}

1. G. Beer, $A$ natural topology for upper semicontinuous functions and a Baire category dual for convergence in measure, Pacific J. Math. 96 (1981), 251-263.

2. U, Upper semicontinuous functions and the Stone Approximation Theorem, J. Approximation Theory 34 (1982), 1-11.

3. E. De Giorgi and T. Franzoni, Su un tipo di convergenza variazionale, Atti Accad. Naz. Lincei Rend. Cl. Sci. Fis. Mat. Natur. (8) 58 (1975), 842-850.

4. S. Dolecki, G. Salinetti and R. Wets, Convergence of functions: equi-semicontinuity, Proc. London Math. Soc. (to appear).

5. M. H. Stone, A generalized Weierstrass approximation theorem, Studies in Modern Analysis, R. C. Buck, ed., M.A.A. Studies in Math., vol. 1, 1962.

6. R. A. Wijsman, Convergence of sequences of convex sets, cones, and functions. II, Trans. Amer. Math. Soc. 123 (1966), 32-45.

Department of Mathematics, California State University, los ANgeles, CALIFORNIA 90032 